\title{
Effects of tumor necrosis factor- $a$ polymorphism on the brain structural changes of the patients with major depressive disorder
}

\author{
Rubai Zhou ${ }^{1,4,7}$, Fan Wang ${ }^{1}$, Guoqing Zhao ${ }^{1,11}$, Weiping Xia ${ }^{1,12}$, Daihui Peng ${ }^{1}$, Ruizhi Mao ${ }^{1}$, Jingjing Xu', \\ Zuowei Wang ${ }^{10}$, Wu Hong ${ }^{1}$, Chen Zhang ${ }^{1}$, Yong Wang ${ }^{1}$, Yousong Su' ${ }^{1}$, Jia Huang ${ }^{1}$, Tao Yang ${ }^{1}$, Jijun Wang ${ }^{3,4,5,6}$, \\ Jun Chen ${ }^{1,2,3}$, Lena Palaniyappan ${ }^{7,8,9}$ and Yiru Fang ${ }^{1,2,3}$
}

\begin{abstract}
Single Nucleotide Polymorphic (SNP) variations of proinflammatory cytokines such as Tumor Necrosis Factor-a (TNF-a) have been reported to be closely associated with the major depressive disorder (MDD). However, it is unclear if proinflammatory genetic burden adversely affects the regional gray matter volume in patients with MDD. The aim of this study was to test whether rs1799724, an SNP of TNF-a, contributes to the neuroanatomical changes in MDD. In this cross-sectional study, a total of 144 MDD patients and 111 healthy controls (HC) well matched for age, sex and education were recruited from Shanghai Mental Health Center. Voxel-based morphometry (VBM) followed by graph theory based structural covariance analysis was applied to locate diagnosis $\times$ genotype interactions. Irrespective of diagnosis, individuals with the high-risk genotype (T-carriers) had reduced volume in left angular gyrus (main effect of genotype). Diagnosis $x$ genotype interaction was exclusively localized to the visual cortex (right superior occipital gyrus). The same region also showed reduced volume in patients with MDD than HC (main effect of diagnosis), with this effect being most pronounced in patients carrying the high-risk genotype. However, neither global nor regional network of structural covariance was found to have group difference. In conclusion, a genetic variation which can increase TNF-a expression selectively affects the anatomy of the visual cortex among the depressed subjects, with no effect on the topographical organization of multiple cortical regions. This supports the notion that anatomical changes in depression are in part influenced by the genetic determinants of inflammatory activity.
\end{abstract}

\section{Introduction}

Major depressive disorder (MDD) is associated with a high rate of morbidity, recurrence, disability and mortality $^{1,2}$. Despite being a leading cause of global disease

Correspondence: Yiru Fang (yirufang@aliyun.com) or

Lena Palaniyappan (Ipalaniy@uwo.ca) or Jun Chen (doctorcj2010@gmail.com) or Jijun Wang (jijunwang27@163.com)

'Division of Mood Disorders, Shanghai Mental Health Center, Shanghai Jiao Tong University School of Medicine, Shanghai, China

${ }^{2}$ CAS Center for Excellence in Brain Science and Intelligence Technology, Shanghai, China

Full list of author information is available at the end of the article. burden ${ }^{3}$, pharmacological strategies of MDD so far have been restricted to the manipulation of monoamine neurotransmission, with only partial effect on the symptom burden.

A growing evidence has suggested that peripheral and central inflammatory response may play an important role in the pathogenesis and development of MDD, especially cytokines ${ }^{4}$. Tumor necrosis factor- $\alpha$ (TNF- $\alpha$ ) is a proinflammatory cytokine produced largely by macrophages ${ }^{5}$, and is involved in both neurotoxic effects as well as neuroprotective effects ${ }^{6}$. Overexpression of TNF- $\alpha$ is

\section{(c) The Author(s) 2018}

(c) Open Access This article is licensed under a Creative Commons Attribution 4.0 International License, which permits use, sharing, adaptation, distribution and reproduction cc) in any medium or format, as long as you give appropriate credit to the original author(s) and the source, provide a link to the Creative Commons license, and indicate if changes were made. The images or other third party material in this article are included in the article's Creative Commons license, unless indicated otherwise in a credit line to the material. If material is not included in the article's Creative Commons license and your intended use is not permitted by statutory regulation or exceeds the permitted use, you will need to obtain permission directly from the copyright holder. To view a copy of this license, visit http://creativecommons.org/licenses/by/4.0/. 
deleterious in the early stage of tissue damage, but it can aid recovery later ${ }^{7}$. Pathophysiologically, elevated TNF- $\alpha$ may arouse lessened neuronal synaptic plasticity, reduced neurotropic factors and declined neurogenesis ${ }^{8,9}$. It has been reported that patients with MDD have enhanced plasma levels TNF- $\alpha$, and antidepressant treatments can decrease this levels ${ }^{10,11}$. Moreover, increasing evidence has shown anti-TNF- $\alpha$ therapy could help to relieve depressive symptoms and repair cognitive impairments ${ }^{12}$. Yang et al. ${ }^{13}$. have shown peripheral TNF- $\alpha$ can affect the brain structure via dendritic elimination independent of central inflammatory activity by using a preclinical mouse model. In particular, inhibition of peripheral TNF- $\alpha$ action prevents structural changes at the synaptic level. The peripherally produced cytokines are also able to act on the brain through deficient blood-brain-barrier, active transport via saturable transport molecules, activating endothelial cells to generate second messengers, and binding to receptors on afferent nerve fibers ${ }^{4,14-16}$.

The rs1799724 $(-850$ or $857 \mathrm{C} / \mathrm{T})$ is a functional single nucleotide polymorphism (SNP) located in the promoter regions of TNF- $\alpha$ gene (GenBank accession no. NG_007462, transcript id NM_000594.3), which can affect the genetic transcriptional activity of $\mathrm{TNF}^{17}$. The high-risk genotype $(-857 \mathrm{C} / \mathrm{T})$ of rs1799724 has been found to give rise to higher levels of mRNA and protein than the low-risk genotype $(-857 \mathrm{C} / \mathrm{C})^{17}$. It has been proved that rs1799724 is an important SNP closely associated with neurological disorders like Alzheimer's disease $^{18,19}$, stroke ${ }^{20}$ and depression after stroke ${ }^{21}$. In the presence of post-stroke depression, serum TNF- $\alpha$ concentration is higher in T-carriers (high-risk genotype) than in non- $\mathrm{T}$ carriers (low-risk genotype), indicating that $805 \mathrm{C} / \mathrm{T}$ genotype moderates serum TNF-a levels especially in the presence of depression ${ }^{21}$.

In recent times, several lines of evidence have implicated cytokines to have neurotrophic functions that help to both promote and prune neuronal synapses, thus serving as experience-dependent plasticity regulators both during development and in adult life ${ }^{22,23}$. In particular, TNF- $\alpha$ has been found critical to the development of unimodal sensory cortex, especially visual ${ }^{24}$, auditory ${ }^{25}$ and somatosensory ${ }^{13}$ cortices. It has been proved by neuroimaging studies that peripheral TNF- $\alpha$ levels are negatively linked to regional volumes in the left occipitotemporal area, left superior occipital gyrus, left inferior parietal lobule and bilateral medial prefrontal cortices of healthy people ${ }^{26}$. Some other SNPs of TNF- $\alpha$ and its receptors have been revealed to affect brain volume of hippocampus, striatum and caudate in healthy people ${ }^{27,28}$.

In this context, we aimed to evaluate the effects of functional TNF- $\alpha$ SNP, rs1799724, on brain structure in both MDD patients and healthy controls using voxel- based morphometry (VBM). We also sought diagnosis $\mathrm{x}$ genotype interaction and followed this up with graph analysis to determine the systemic impact of any localized changes.

\section{Materials and methods \\ Participants}

According to a previous study ${ }^{29}$, the minimum sample size in each group should be 80 to guarantee $80 \%$ voxelbased power for grey matter analysis, and 144 patients with MDD and 111 healthy controls were included in this study finally. The patients, who met Diagnostic and Statistical Manual of Mental Disorders (DSM)-IV-TR criteria for MDD, were recruited from the clinics of Shanghai Mental Health Center (SMHC). They were 20-40 years old, right-handed, and educated up to or above junior high school level. All patients were medication-free when they were scanned, including 80 drug-naïve first-episode patients, 27 drug-naïve multiepisode patients, and 37 multi-episode patients who were not medicated for at least one month before the scanning. Moreover, when assessed by Hamilton's Depression Scale with 17 items, the patients included should be with total scores no less than 17 and scores of depressive mood item no less than 2. Those patients with other axes I disorders and debilitating general medical disorders were excluded. Age, sex, and education matched $\mathrm{HCs}$, who could pass our screening and had no history of psychosis or debilitating general medical disorders, were also recruited. This study was approved by the Institutional Review Board of Shanghai Mental Health.

Centre after strict inspection of the protocol, and all the participants have written informed consents. (Clinical Trial Registry Number: NCT01764867, http://www. clinicaltrials.gov)

\section{Genotyping}

The genomic DNA was isolated from the peripheral blood using Lifefeng Blood DNA Kit (Shanghai Lifefeng Biotech Co., Ltd, China). The SNP rs1799724 of TNF- $\alpha$ was genotyped via the Multiplex SnaPshot technique (Genesky Biotechnologies, Inc., Shanghai, China). The PCR primer was rs9964_rs9724F/R. All the 255 subjects were successfully genotyped for this promoter polymorphism of TNF- $\alpha$. Hardy-Weinberg equilibrium analysis for the single SNP was performed using Chi-squared Test with SPSS19.0 (SPSS Inc., Chicago, IL, USA). The SNP rs1799724 genotype distribution of all the subjects was in Hardy-Weinberg equilibrium $(p=0.806)$.

\section{Magnetic resonance imaging (MRI)}

All the MRI data were obtained in the radiology department of SMHC using a 3.0 T Siemens scanner and 
high-resolution T1 images were used in the analyses of the present study. Three-dimensional magnetization prepared rapid acquisition gradient echo sequence (3DMPRAGE) had repetition time $=2530 \mathrm{~ms}$, echo time $=$ $3.65 \mathrm{~ms}$, flip angle $\alpha=7^{\circ}, 224$ sagittal slices, field of view $=256 \times 256 \mathrm{~mm}$, matrix $=256 \times 256$, thickness $=1 \mathrm{~mm}$. Images were checked out for movement artifacts and homogeneity of image intensity immediately after scans and repeated if necessary. We also collected a clinical diagnostic MR sequence in the same session and excluded individuals who had ischemia, cavum septum pellucidum, radiologically notable cerebral leukomalacia as determined by a radiologist.

\section{Voxel-based morphometry (VBM)}

We used VBM8 ${ }^{30}$ (Department of Psychiatry, University of Jena) toolbox based on Statistical Parametric Mapping (SPM8, Institute of Neurology, UK) and Matlab (Mathworks, USA). First, we reoriented the images manually to set their origins to the anterior commissure. Then the T1 image of every subject was segmented into gray matter (GM), white matter (WM) and cerebrospinal fluid $(\mathrm{CSF})^{31}$. Meanwhile, the total volume of GM, WM, and CSF were estimated and reported by VBM8. After that, a high-dimensional non-linear DARTEL (Diffeomorphic Anatomical Registration Through Exponentiated $\mathrm{Lie}^{32}$ ) algebra was performed for normalization and modulation of the GM and WM. The modulated GM maps were smoothed with an $8 \mathrm{~mm}$ full-width at half maximum Gaussian kernel.

VBM statistics were conducted in SPM8 using a $2 \times 2$ full factorial model defining diagnosis (HC; MDD) and genotype (low risk; high risk) as two factors, while using age, sex, years of education and total intracranial volume as covariates to remove their effects on variance. An explicit grey matter mask was used to prevent effects occurring outside grey matter. We assessed the main effect of diagnosis, the main effect of genotype and the interaction effect, the disease effects within low-risk or high-risk subgroup using $\mathrm{F}$ contrasts. All the significant results at $p<0.001$ (uncorrected) were reported. With $\mathrm{DPABI}^{33}$ software, AlphaSim program based on Monte Carlo simulation was applied for multiple comparison corrections ${ }^{34}$, and the current version has solved the bug raised by Eklund et $\mathrm{al}^{35}$ with the real smoothness in the three directions calculated from the residuals. 5000 simulations for every statistic map were conducted and strict threshold was applied (voxel-wise $p<0.001$ and cluster-wise $p<0.05$, two-tailed). Anatomical regions emerging from VBM analysis were labeled according to Anatomical Automatic Labeling (AAL) atlas and visualized with BrainNet Viewer ${ }^{36}$. Additionally, we used xjView toolbox (http://www.alivelearn.net/xjview) together with Easy Volume toolbox (http://www.sbirc.ed.ac.
uk/LCL/LCL_M1.html) to extract mean volumes of the clusters that had shown significant effects in VBM analysis.

\section{Graph theory analysis}

We primarily applied Graph Analysis Toolbox (GAT ${ }^{37}$ ) to implement graph analysis of the structural networks of MDD patients and HCs in the high-risk subgroup. First, 90 regions of interest (ROIs) based on the cerebral part of Anatomical Automatic Labeling (AAL) atlas were defined, masks of them were generated using WFU PickAtlas Toolbox $^{38}$ and were resliced to the same dimension as modulated, normalized GM images from VBM preprocessing step. REX code (http://web.mit.edu/swg/ software.htm) was used to extract the mean volume of every ROI. Second, the extracted volume data of 90 ROIs were used to construct structural correlation networks. A $90 \times 90$ correlation matrix (R) was generated in each group with each entry $r_{i j}$ representing the Pearson correlation coefficient between the volume of ROI $i$ and $j$. Then each $\mathrm{R}$ matrix was converted to a binary association matrix (A), with entry $\mathrm{a}_{\mathrm{ij}}$ considered 1 If $r_{i j}$ bigger than a specific threshold and zero otherwise. Thresholding and construction of structural networks were carried out at a range of network densities ( $D_{\text {min }}$ : 0.24: 0.02: 0.5), across which also group comparison was conducted. The lower limit of the range was the calculated minimum density allowing all nodes to be fully connected with each other, and the upper limit of the range was set to 0.5 , as densities above $50 \%$ are not sparse anymore and thus not biologically meaningful for brain networks. Accordingly, a graph (G) including 90 nodes was derived from $\mathrm{A}$, and regions $\mathrm{i}$ and $j$ were regarded as connected if $g_{i j}$ is unity. Network degree (E) was equal to the number of edges, and the network density (D) was used to present the fraction of actually existent edges to all possible links. Third, topology of the global network, such as small-worldness, normalized clustering and normalized path length, were measured using codes in the Brain Connectivity Toolbox $^{39}$ at both the network and regional level. The smallworldness of a network has two key measurements, clustering coefficient $(C)$ and characteristic path length (L). The clustering coefficient is a measure of network segregation and is represented as the mean value of clustering coefficients, while the path length is a measure of network integration and is defined as the mean shortest path length between all pairs of nodes in the network. All the measurements were compared to the consistent mean values of a benchmark random graph so as to appraise the topology of the brain network, and $\mathrm{m}$ (the number) null networks were generated for normalization of $\mathrm{C}$ and $\mathrm{L}$. Normalized $\mathrm{C}$ and normalized $\mathrm{L}$ were calculated as $\mathrm{C} /$ $\mathrm{C}_{\text {rand }}$ and $\mathrm{L} / \mathrm{L}_{\text {rand }}$, and small-wordness was obtained as $\left[\mathrm{C} / \mathrm{C}_{\text {rand }}\right] /\left[\mathrm{L} / \mathrm{L}_{\text {rand }}\right] \quad\left(\mathrm{C}_{\text {rand }}\right.$ and $\mathrm{L}_{\text {rand }}$ were the mean 
Table 1 Demographics of MDD patients and HCs

\begin{tabular}{|c|c|c|c|c|c|}
\hline & & MDD & $\mathrm{HC}$ & $X^{2} /$ Mann-Whitney U/t & $p$ value \\
\hline Male & & $60(41.7 \%)$ & $53(47.7 \%)$ & 0.939 & 0.332 \\
\hline Age & & $28.17 \pm 5.91$ & $27.63 \pm 5.43$ & 7548.000 & 0.446 \\
\hline Years of education & & $15.29 \pm 2.55$ & $15.51 \pm 2.95$ & 7233.500 & 0.188 \\
\hline Total brain volume (ml) & & $1410.42 \pm 132.17$ & $1429.71 \pm 126.45$ & -1.177 & 0.240 \\
\hline \multirow[t]{2}{*}{ rs1799724 } & CC & $110(76.4 \%)$ & $83(74.8 \%)$ & 0.089 & 0.766 \\
\hline & $\mathrm{CT} / \mathrm{TT}$ & $34(23.6 \%)$ & $28(25.2 \%)$ & & \\
\hline
\end{tabular}

clustering coefficient and the characteristic path length of the $\mathrm{m}$ random networks.). Compared to random network, small-world network is characterized by significantly higher clustering coefficient $\left(\mathrm{C} / \mathrm{C}_{\text {rand }}\right.$ ratio greater than 1$)$ as well as similar characteristic path length $\left(\mathrm{L} / \mathrm{L}_{\text {rand }}\right.$ ratio close to 1). In terms of regional network characteristics, nodal clustering, degree, and betweenness were estimated and compared at the minimal density for significant brain regions from VBM analyses. Nodal clustering quantifies how close its neighbors are to being a clique, nodal degree is defined as the number of the edges from a node to others, and nodal betweenness is represented as the proportion of all shortest paths in the network that pass through a given node. The regional measures were normalized by the mean network value respectively, and then compared between groups. Forth, the within-group difference of network topology was identified, the betweengroup difference of global and regional network measures were tested using non-parametric permutation test with 1000 repetitions.

The VBM and GAT analyses were repeated more than twice with the same results.

\section{Statistical analysis}

Statistical analyses of clinical data were conducted with SPSS19.0 (SPSS Inc., Chicago, IL, USA). Normal distribution test was performed using One-Sample Kolmogorov-Smirnov Test in groups of MDD and $\mathrm{HC}$ separately, and Levene's Tests were used to test the homogeneity of variances. (Table S1 in the Supplementary Materials.) The differences between the MDD and HC were evaluated using Mann-Whitney Test (age and years of education, which did not coincide with normal distribution), two-sample $t$-Test (total brain volume) and Chi-square test (gender and genotype).

\section{Results}

\section{Demographics}

No significant difference of age, gender, years of education, total brain volume or distribution of SNP genotypes was found between groups of MDD and HC (Table 1).

\section{VBM analysis}

The interactive effects of diagnosis and rs1799724 primarily influenced right superior occipital gyrus, the orbital part of right middle frontal gyrus and the triangular part of the right inferior frontal gyrus.(Table 2) After AlphaSim correction, however, diagnosis $\mathrm{x}$ genotype interaction was exclusively localized to the visual cortex (right superior occipital gyrus, Fig. 1), and the same region also showed reduced volume in patients with MDD than HC (main effect of diagnosis, Table 2, Fig S1 in the Supplementary Materials). The effect of rs1799724 mainly located in brain regions of left angular gyrus, left middle occipital gyrus, the medial part of left superior frontal gyrus, right supplementary motor area and left precuneus. (Table 2) Among these regions, left angular gyrus was focalized after correction for multiple comparisons, with high-risk individuals showing reduced volume (Fig S2 in the Supplementary Materials). From post-hoc T-Test, low-risk MDD revealed decreased volume in left inferior parietal gyrus $\left(p_{\text {Alphasim }}<0.05\right)$, left angular gyrus, right precuneus gyrus, left inferior parietal gyrus and left supplementary motor area than low-risk HC (Table 2, Fig S3 in the Supplementary Materials), while depressive individuals showed less volume in right superior occipital gyrus $\left(p_{\text {AlphaSim }}<0.05\right)$ and the orbital part of right middle frontal gyrus than $\mathrm{HC}$ in the high-risk subgroup (Table 2, Fig S4 in the Supplementary Materials).

\section{GAT analysis in the high-risk subgroup}

The global network topology of shortest path length, clustering coefficient and small-worldness were measured across a range of densities (Dmin: 0.24: 0.02: 0.5) and compared within and between groups. In each group of high-risk MDD and $\mathrm{HC}$, characteristic path length approached to 1, while clustering coefficient and smallworldness were higher than 1, indicating a small-world organization (Fig. 2). However, no significant difference of clustering coefficient, shortest path length or smallworldness was found between the two groups of highrisk MDD and $\mathrm{HC}$, as all the difference values appeared within the confidence interval in Fig. 3. Regional network 
Table 2 Significant brain regions in VBM analysis $(p<0.001$, uncorrected)

\begin{tabular}{|c|c|c|c|c|c|c|c|}
\hline Anatomical region & Cluster size & $F$ value & $\begin{array}{l}\text { Low-risk HC } \\
\left(\mathrm{mm}^{3}\right)\end{array}$ & $\begin{array}{l}\text { High-risk HC } \\
\left(\mathrm{mm}^{3}\right)\end{array}$ & $\begin{array}{l}\text { Low-risk MDD } \\
\left(\mathrm{mm}^{3}\right)\end{array}$ & $\begin{array}{l}\text { High-risk MDD } \\
\left(\mathrm{mm}^{3}\right)\end{array}$ & MNI coordinates \\
\hline \multicolumn{8}{|c|}{ Interaction of diagnosis and rs 1799724} \\
\hline Right superior occipital gyrus ${ }^{a}$ & 174 & 20.57 & $357.9 \pm 125.9$ & $437.3 \pm 116.1$ & $364.4 \pm 113.8$ & $304.6 \pm 101.9$ & $22.5,-82.5,22.5$ \\
\hline $\begin{array}{l}\text { Right middle frontal gyrus, } \\
\text { orbital part }\end{array}$ & 14 & 13.55 & $28.9 \pm 8.7$ & $32.5 \pm 9$ & $31.1 \pm 10$ & $23.2 \pm 10.1$ & $24,57,-18$ \\
\hline $\begin{array}{l}\text { Right inferior frontal gyrus, } \\
\text { triangular part }\end{array}$ & 15 & 11.79 & $23.5 \pm 6.1$ & $18.8 \pm 5.4$ & $20.9 \pm 5.3$ & $22.3 \pm 6.4$ & $54,33,7.5$ \\
\hline \multicolumn{8}{|l|}{ Main effect of diagnosis } \\
\hline Right superior occipital gyrus ${ }^{\mathrm{a}}$ & 124 & 19.91 & $261.4 \pm 100.5$ & $323.2 \pm 92$ & $262.1 \pm 90.4$ & $216 \pm 83.7$ & $19.5,-85.5,21$ \\
\hline \multicolumn{8}{|l|}{ Main effect of rs1799724 } \\
\hline Left angular gyrus $^{a}$ & 526 & 20.07 & $528.8 \pm 107.6$ & $451.9 \pm 88.4$ & $488 \pm 96$ & $438.7 \pm 116.4$ & $-40.5,-66,57$ \\
\hline Left middle occipital gyrus & 23 & 15.23 & $32.6 \pm 15.6$ & $44.1 \pm 17.5$ & $34.7 \pm 15.7$ & $44.1 \pm 17.9$ & $-13.5,-88.5,-6$ \\
\hline Left middle occipital gyrus & 17 & 12.16 & $0.2 \pm 0.3$ & $0.1 \pm 0.1$ & $0.2 \pm 0.2$ & $0.1 \pm 0.2$ & $-33,-97.5,19.5$ \\
\hline $\begin{array}{l}\text { Left superior frontal gyrus, } \\
\text { medial part }\end{array}$ & 62 & 12.10 & $77 \pm 15.8$ & $67.4 \pm 18.5$ & $72.4 \pm 18$ & $65.4 \pm 18.2$ & $-1.5,28.5,43.5$ \\
\hline $\begin{array}{l}\text { Right supplementary motor } \\
\text { area }\end{array}$ & 20 & 12.40 & $26.1 \pm 8.1$ & $19.8 \pm 9.6$ & $22.4 \pm 9.2$ & $21.1 \pm 9.2$ & $3,-19.5,51$ \\
\hline Left precuneus & 49 & 13.53 & $17.3 \pm 13.1$ & $12 \pm 9.3$ & $15.7 \pm 11.5$ & $13.6 \pm 11.7$ & $-3,-46.5,66$ \\
\hline \multicolumn{8}{|l|}{ Disease effects in low-risk subgroup } \\
\hline Left inferior parietal gyrus ${ }^{\mathrm{a}}$ & 286 & 17.37 & $440.1 \pm 80.3$ & $405.1 \pm 84.7$ & $398.2 \pm 73.7$ & $383 \pm 75.3$ & $-42,-51,51$ \\
\hline Left angular gyrus & 84 & 14.45 & $204.1 \pm 51$ & $178.1 \pm 58$ & $180.9 \pm 50.1$ & $183 \pm 57.6$ & $-46.5,-55.5,33$ \\
\hline Right precuneus gyrus & 83 & 13.85 & $187.2 \pm 45.4$ & $165.6 \pm 38.9$ & $159.4 \pm 44.2$ & $171.7 \pm 40.3$ & $12,-58.5,43.5$ \\
\hline Left inferior parietal gyrus & 62 & 14.26 & $57.5 \pm 14.2$ & $51.3 \pm 9.1$ & $50.8 \pm 12$ & $53.3 \pm 11.5$ & $-58.5,-45,51$ \\
\hline Left supplementary motor area & 14 & 12.32 & $6.8 \pm 5.4$ & $5.2 \pm 4.4$ & $5.2 \pm 5.3$ & $4.7 \pm 4.1$ & $1.5,10.5,45$ \\
\hline \multicolumn{8}{|c|}{ Disease effects in high-risk subgroup } \\
\hline Right superior occipital gyrus ${ }^{a}$ & 227 & 25.39 & $449.8 \pm 145$ & $543 \pm 142.8$ & $455.1 \pm 132.2$ & $384.6 \pm 115.8$ & $21,-84,22.5$ \\
\hline $\begin{array}{l}\text { Right middle frontal gyrus, } \\
\text { orbital part }\end{array}$ & 24 & 14.14 & $49.9 \pm 12.8$ & $54.3 \pm 12.5$ & $51.8 \pm 14.8$ & $40.6 \pm 16.8$ & $25.5,55.5,-18$ \\
\hline
\end{tabular}

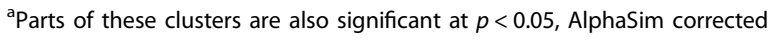

measures of right superior occipital gyrus, left angular gyrus and left inferior parietal gyrus were compared at the minimal density $(0.24)$, nevertheless, no significant difference of nodal clustering, degree or betweenness was found between groups (Table S1 in the Supplementary Materials).

\section{Discussion}

In the present study, we report three major findings. Firstly, TNF polymorphism (rs1799724) has a predominant effect on the structure of the visual cortex in the presence of depression. Secondly, as there is no notable effect on the structural covariance networks, it is likely that the morphological aberration is late in onset and highly localized in nature. Thirdly, in medication-free
MDD patients, grey matter changes are of small effect size and limited in distribution ${ }^{40}$.

Our findings demonstrate that a polymorphism, which is potentially linked to higher transcription of TNF- $\alpha$, is associated with structural changes in the occipital cortex, especially in the presence of depression. These results add to the burgeoning literature implicating visual stimulus processing deficits ${ }^{41}$ and occipital cortex abnormalities in $\mathrm{MDD}^{42}$. While gray matter reduction in occipital cortex has been reported inconsistently in $\mathrm{MDD}^{43-45}$, prior reports highlight reduced functional connectivity ${ }^{46-51}$, perfusion deficits ${ }^{52,53}$ and abnormal structure $^{54-56}$ of occipital cortex in MDD. Given that our sample comprised largely of drug-naïve first episode MDD, and the fact that only high-risk group had the MDD-related 
reduction in occipital cortex volume, this association is unlikely to be explained by the use of antidepressants or duration of illness.

The $\mathrm{T}$ allele of rs1799724 has the higher transcriptional activity for $\mathrm{TNF}^{17}$. Higher levels of TNF- $\alpha^{57}$ relates to depression-like symptoms ${ }^{58}$, thus indicating that the

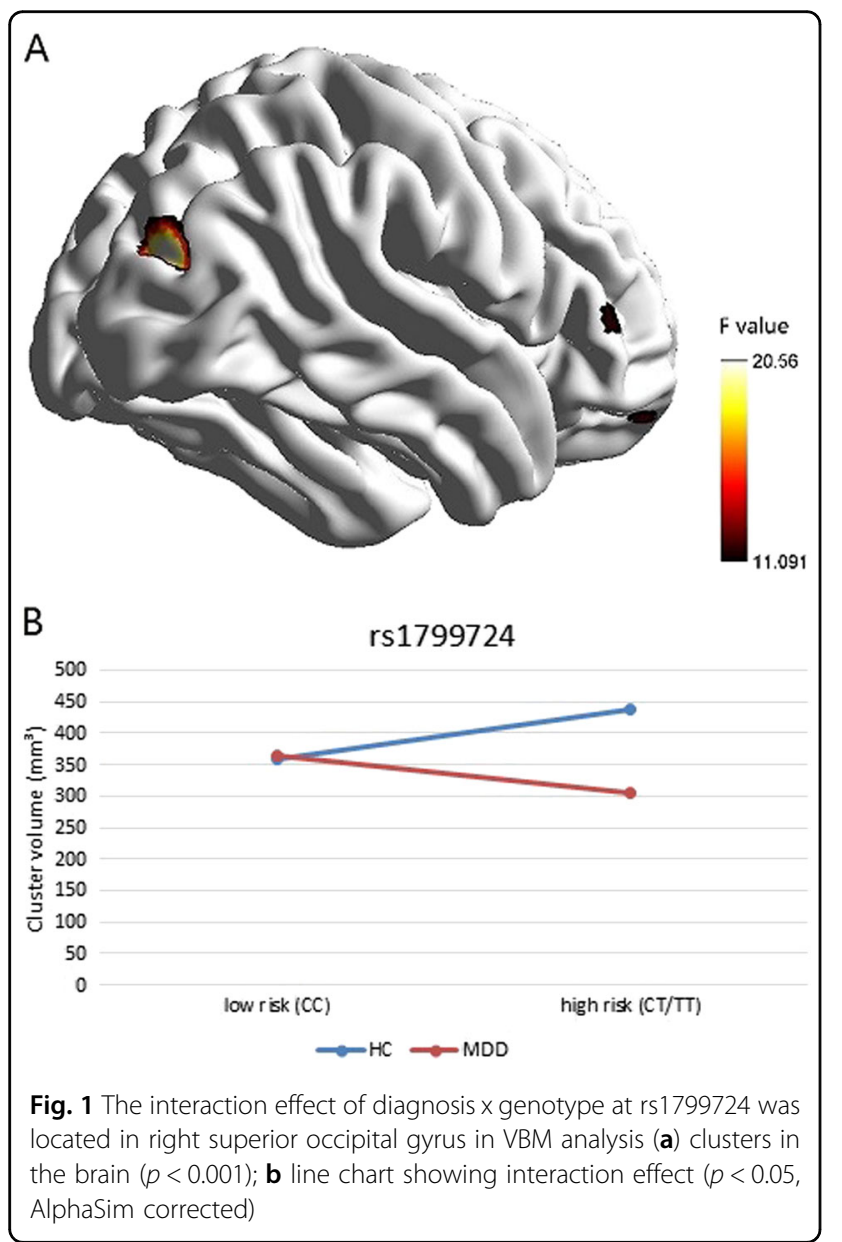

carriers of $\mathrm{T}$ form a high-risk group. Of interest to our findings, TNF- $\alpha$ has been showed to play an important role in the synaptic plasticity to contribute to the anatomy of visual cortex. In particular, in the absence of TNF- $\alpha$, compensatory adjustment to monocular deprivation does not occur in the non-deprived eye ${ }^{59}$. TNF- $\alpha$ has been shown to mediate experience-dependent plasticity through homeostatic synaptic scaling ${ }^{24}$ as well as longterm potentiation ${ }^{59}$. Of further relevance to depression where diurnal variation in emotion, cognition, and motivation occurs in a prominent fashion, the notable diurnal variation has been reported in the expression of TNF- $\alpha$ receptors in the murine visual cortex, as a response to daylight ${ }^{60}$. As the visual system is used differently in day and night, the levels of some cytokines, including TNF- $\alpha$, and the number of their receptor cells varies in different time of day ${ }^{60}$. TNF- $\alpha$ expression and receptor cells increased in the dark compared to the light. The alteration in visual cortex and related emotion and cognition of MDD patients may be explained by varying TNF- $\alpha$ and other cytokines partly. Taken together, our observation of MDD $\times$ genotype interaction indicates that in a subgroup of depressed subjects with the high-risk variant, the trophic effects of TNF- $\alpha$ on visual cortex may be disrupted.

We studied structural covariance using graph theory, to detect the effect of TNF polymorphism on the coordinated maturation of distributed, large-scale morphometric networks. We observed no MDD $\times$ genotype interaction on the structural covariance across the entire brain. Morphometric networks with tightly co-varying patterns of brain anatomy are likely to have mutually shared trophic influences such as functional co-activation or expression of plasticity modulating factors ${ }^{61}$. The lack of disruption in structural covariance in depressed subjects with the high-risk genotype suggests that the effect of TNF- $\alpha$ may be either temporally restricted (e.g.,

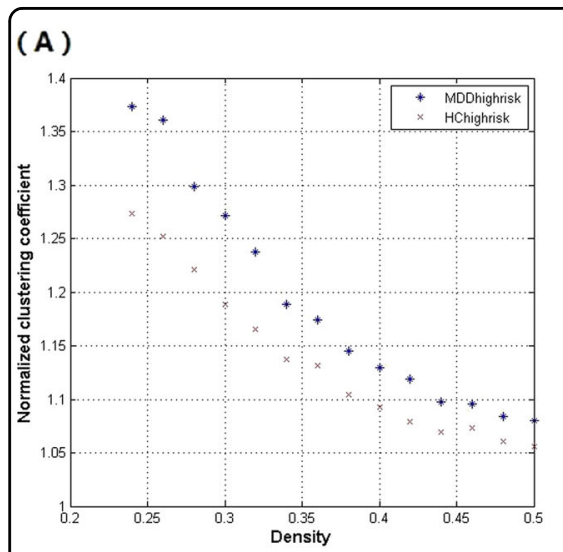

(B)

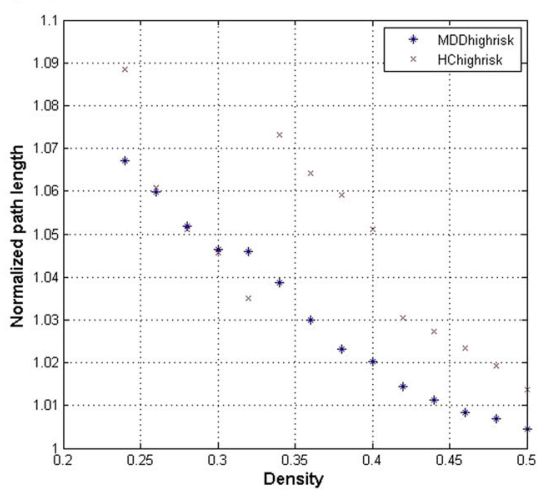

(C)

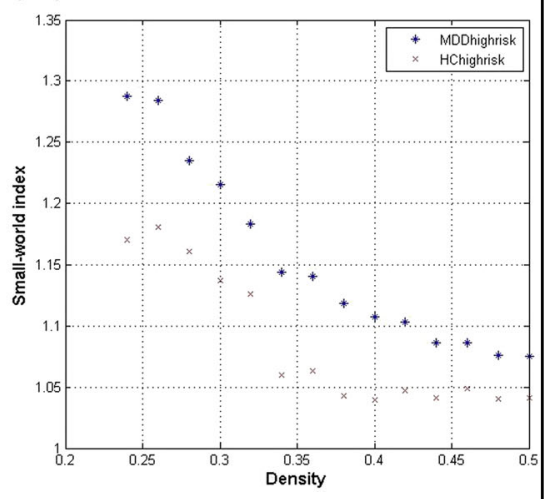

Fig. 2 The global network is measured at different network density. Normalized clustering (a), normalized path length (b), and small-world index (c) of the major depressive disorder (MDD) and healthy control (HC) networks in the high-risk subgroup 


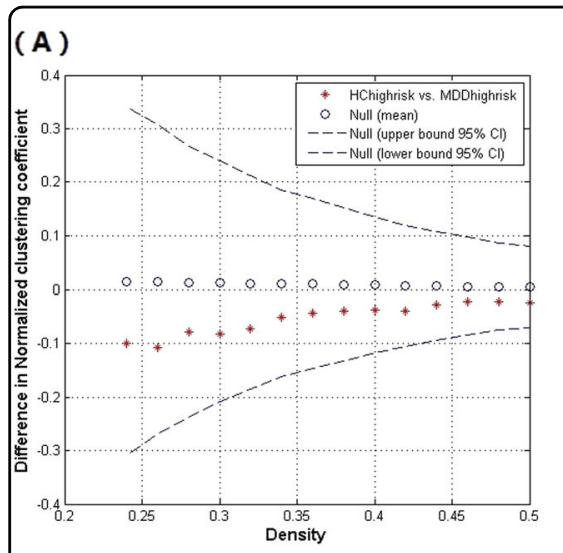

(B)

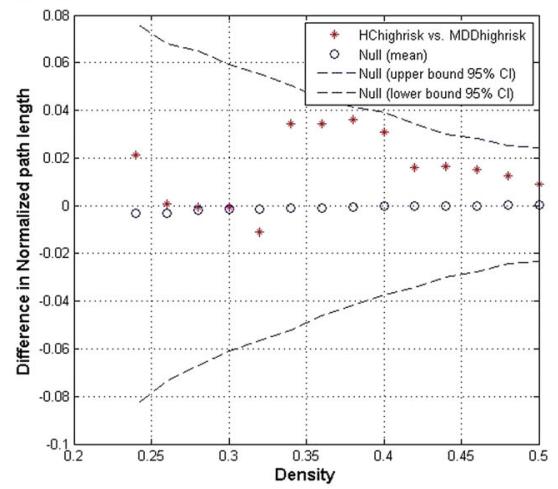

( C)

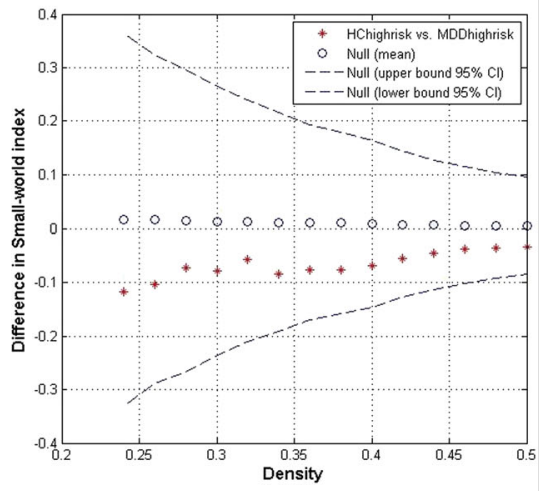

Fig. 3 The global network differences between major depressive disorder (MDD) and healthy control (HC) participants in the high-risk subgroup at different network density. The $95 \%$ confidence intervals $(\mathrm{Cl})$ and group differences in normalized clustering (a), normalized path length (b), and small-world index (c). The * marker shows the difference between the high-risk subgroup of MDD and HC; the * signs falling outside of the confidence intervals indicate the densities in which the difference is significant at $p<0.05$. The positive values show $H C_{\text {highrisk }}>M_{D D}$ highrisk and negative values show $H C_{\text {highrisk }}<M D D_{\text {highrisk }}$

periods of inflammatory activity or critical window of development) and/or spatially restricted to the occipital cortex. It is important to consider this in light of our observation that there was a small, statistically insignificant effect of diagnosis $x$ genotype interaction in other brain regions in our sample. Such spatially limited grey matter deficits are in line with several VBM studies investigating a comparable sample (first episode, unmedicated subjects with MDD $)^{40}$.

There are several limitations that need to be considered when interpreting the results of this study. Firstly, as a transcriptional regulator, rs 1799724 is not the only SNPs in the promoter region of TNF- $\alpha$; nevertheless, studies have suggested a link between rs1799724 and neurodegenerative/neurovascular disorders such as Alzheimer's disease $^{18,19}$, stroke ${ }^{20}$ and depression after stroke ${ }^{21}$. We plan to undertake further neuroimaging studies with other SNPs of TNF- $\alpha$, subsequent to appropriate approvals to study their individual effects as well as interactions among the various polymorphic variants. Secondly, we lacked functional data on task performance (especially for visual stimulation) and neuropsychological data relevant to visual processing, to further study the implications of the observed structural change. This is one of our goals for the future studies. Thirdly, it is possible that there are other potential confounding factors mediating the observed relationship between TNF polymorphism and brain structure in depression. We lacked sufficient quantitative data on smoking, alcohol and drug use, stress and childhood trauma, that may also influence the brain structure.

In conclusion, the TNF- $\alpha$ SNP, rs1799724 (TNF-850 or $857 \mathrm{C} / \mathrm{T}$ ), has a notable effect on the brain structure in both patients with MDD and healthy controls, with disease-related effects localizable to the occipital cortex.
Our observations raise questions regarding the disruptions in the trophic role played by pro-inflammatory cytokine system in depression. Our results are highly relevant to understanding the brain-basis of antiinflammatory treatments in depression, as well as isolating sub-groups of depressed subjects who may differ in cortical anatomy, genotype as well as response to novel therapeutics.

\section{Acknowledgements}

This work was supported by National Key R\&D Program of China (2016YFC1307100), National Natural Science Foundation of China (91232719, 81201056, 81301159, 81771465), The "12th Five-year Plan" of National Key Technologies R\&D Program (2012BAI01B04). This work was also supported by the Bucke Family Fund and the Academic Medical Organization of South Western Ontario (LP).

\section{Author details}

'Division of Mood Disorders, Shanghai Mental Health Center, Shanghai Jiao Tong University School of Medicine, Shanghai, China. ${ }^{2}$ CAS Center for Excellence in Brain Science and Intelligence Technology, Shanghai, China. ${ }^{3}$ Shanghai Key Laboratory of Psychotic disorders, Shanghai, China.

${ }^{4}$ Department of EEG \& Neuroimaging, Shanghai Mental Health Center, Shanghai Jiao Tong University School of Medicine, Shanghai, China. ${ }^{5}$ Bio-X Institutes, Key Laboratory for the Genetics of Developmental and Neuropsychiatric Disorders (Ministry of Education), Shanghai, China. ${ }^{6}$ Brain Science and Technology Research Center, Shanghai Jiao Tong University, Shanghai, China. ${ }^{7}$ Robarts Research Institute\& The Brain and Mind Institute, University of Western Ontario, London, ON, Canada. ${ }^{8}$ Department of Psychiatry, University of Western Ontario, London, ON, Canada. 'Lawson Health Research Institute, London, ON, Canada. ${ }^{10} \mathrm{Hongkou}$ District Mental Health Center of Shanghai, Shanghai, China. "'Department of Psychology, Provincial Hospital Affiliated to Shandong University, Jinan 250021, China. ${ }^{12}$ Department of Medical Psychology, Xinhua Hospital, Shanghai Jiao Tong University School of Medicine, Shanghai, China

\section{Conflict of interest}

LP reports personal speaker/advisory fees from Otsuka Canada, Janssen Canada, SPMM Course Limited, UK, Canadian Psychiatric Association; book royalties from Oxford University Press; investigator-initiated educational grants from Janssen Canada, Otsuka Canada outside the submitted work. The rest of the authors declare that they have no conflict of interest. 


\section{Publisher's note}

Springer Nature remains neutral with regard to jurisdictional claims in published maps and institutional affiliations.

Supplementary Information accompanies this paper at (https://doi.org/ 10.1038/s41398-018-0256-x).

Received: 20 March 2018 Revised: 30 July 2018 Accepted: 5 August 2018 Published online: 11 October 2018

\section{References}

1. Demyttenaere, K. et al. Prevalence, severity, and unmet need for treatment of mental disorders in the World Health Organization World Mental Health Surveys. Jama 291, 2581-2590 (2004).

2. Phillips, M. R., Li, X. \& Zhang, Y. Suicide rates in China, 1995-99. Lancet 359 835-840 (2002).

3. Lopez, A. D., Mathers, C. D., Ezzati, M., Jamison, D. T. \& Murray, C. J. L. Global and regional burden of disease and risk factors, 2001: systematic analysis of population health data. Lancet 367, 1747-1757 (2006).

4. Miller, A. H., Maletic, V. \& Raison, C. L. Inflammation and its discontents: the role of cytokines in the pathophysiology of major depression. Biol. Psychiatry $\mathbf{6 5}$, 732-741 (2009).

5. Wajant, H., Pfizenmaier, K. \& Scheurich, P. Tumor necrosis factor signaling. Cell Death Differ. 10, 45-65 (2003).

6. Sriram, K. \& O'Callaghan, J. P. Divergent roles for tumor necrosis factor-alpha in the brain. J. Neurolmmune Pharmacol. 2, 140-153 (2007).

7. Stellwagen, D. The contribution of TNFalpha to synaptic plasticity and nervous system function. Adv. Exp. Med. Biol. 691, 541-557 (2011).

8. Hashmi, A. M., Butt, Z. \& Umair, M. Is depression an inflammatory condition? A review of available evidence. J. Pak. Med. Assoc. 63, 899-906 (2013).

9. Santello, M. \& Volterra, A. TNFalpha in synaptic function: switching gears. Trends Neurosci. 35, 638-647 (2012).

10. Ma, K., Zhang, H. \& Baloch, Z. Pathogenetic and therapeutic applications of tumor necrosis factor-alpha (TNF-alpha) in major depressive disorder: a systematic review. Int. J. Mol. Sci. 17, 733 (2016).

11. Hannestad, J., DellaGioia, N. \& Bloch, M. The effect of antidepressant medication treatment on serum levels of inflammatory cytokines: a meta-analysis. Neuropsychopharmacology. 36, 2452-2459 (2011).

12. Bortolato, B., Carvalho, A. F., Soczynska, J. K., Perini, G. I. \& Mclntyre, R. S. The involvement of TNF-alpha in cognitive dysfunction associated with major depressive disorder: an opportunity for domain specific treatments. Curr. Neuropharmacol. 13, 558-576 (2015).

13. Yang, G., Parkhurst, C. N., Hayes, S. \& Gan, W. B. Peripheral elevation of TNFalpha leads to early synaptic abnormalities in the mouse somatosensory cortex in experimental autoimmune encephalomyelitis. Proc. Natl Acad. Sci. USA 110, 10306-10311 (2013).

14. Eyre, H. \& Baune, B. T. Neuroplastic changes in depression: a role for the immune system. Psychoneuroendocrinology 37, 1397-1416 (2012).

15. Kubera, M., Obuchowicz, E., Goehler, L., Brzeszcz, J. \& Maes, M. In animal models, psychosocial stress-induced (neuro)inflammation, apoptosis and reduced neurogenesis are associated to the onset of depression. Progress. Neuro Psychopharmacol. Biol. Psychiatry 35, 744-759 (2011).

16. Dantzer, R., O'Connor, J. C., Freund, G. G., Johnson, R. W. \& Kelley, K. W. From inflammation to sickness and depression: when the immune system subjugates the brain. Nat. Rev. Neurosci. 9, 46-56 (2008).

17. Kimura, K., Takayanagi, R., Yokoyama, H. \& Yamada, Y. Effects of tumor necrosis factor alpha-857C/T polymorphism on the expression of tumor necrosis factor alpha. APMIS 124, 669-674 (2016).

18. Di Bona, D. et al. Systematic review by meta-analyses on the possible role of TNF-alpha polymorphisms in association with Alzheimer's disease. Brain Res. Rev. 61, 60-68 (2009).

19. Laws, S. M. et al. TNF polymorphisms in Alzheimer disease and functional implications on CSF beta-amyloid levels. Human. Mutat. 26, 29-35 (2005).

20. Park, H. J., Kim, S. K., Park, H. K. \& Chung, J. H. Association of promoter polymorphism $-857 C / T$ (rs1799724) in tumor necrosis factor gene with intracerebral hemorrhage in Korean males. Neurol. Res. 39, 90-95 (2017).

21. Kim, J. M. et al. Associations of tumor necrosis factor-alpha and interleukin1 beta levels and polymorphisms with post-stroke depression. Am. J. Geriatr. Psychiatry 25, 1300-1308 (2017).
22. Deverman, B. E. \& Patterson, P. H. Cytokines and CNS development. Neuron 64, 61-78 (2009).

23. Alboni, S. \& Maggi, L. Editorial: cytokines as players of neuronal plasticity and sensitivity to environment in healthy and pathological brain. Front. Cell. Neurosci. 9, 508 (2015).

24. Kaneko, M., Stellwagen, D., Malenka, R. C. \& Stryker, M. P. Tumor necrosis factoralpha mediates one component of competitive, experience-dependent plasticity in developing visual cortex. Neuron 58, 673-680 (2008).

25. Yang, S., Zhang, L. S., Gibboni, R., Weiner, B. \& Bao, S. Impaired development and competitive refinement of the cortical frequency map in tumor necrosis factor-alpha-deficient mice. Cereb. cortex 24, 1956-1965 (2014).

26. Zhang, $H$. et al. The relationship between inflammatory markers and voxelbased gray matter volumes in nondemented older adults. Neurobiol. Aging 37, 138-146 (2016).

27. Stacey, D. et al. TNF receptors 1 and 2 exert distinct region-specific effects on striatal and hippocampal grey matter volumes (VBM) in healthy adults. Genes, brain, Behav. 16, 352-360 (2017).

28. Baune, B. T. et al. Tumor necrosis factor gene variation predicts hippocampus volume in healthy individuals. Biol. Psychiatry 72, 655-662 (2012).

29. Suckling, J. et al. Power calculations for multicenter imaging studies controlled by the false discovery rate. Human. Brain Mapp. 31, 1183-1195 (2010).

30. Ashburner, J. \& Friston, K. J. Voxel-based morphometry-the methods. Neurolmage 11(6 Pt 1), 805-821 (2000).

31. Ashburner, J. \& Friston, K. J. Unified segmentation. Neurolmage 26, 839-851 (2005).

32. Ashburner, J. A fast diffeomorphic image registration algorithm. Neurolmage 38, 95-113 (2007).

33. Yan, C. G., Wang, X. D., Zuo, X. N. \& Zang, Y. F. DPABI: Data Processing \& Analysis for (Resting-State) Brain Imaging. Neuroinformatics 14, 339-351 (2016).

34. Ledberg, A., Akerman, S. \& Roland, P. E. Estimation of the probabilities of 3D clusters in functional brain images. Neurolmage 8, 113-128 (1998).

35. Eklund, A., Nichols, T. E. \& Knutsson, H. Cluster failure: why fMRI inferences for spatial extent have inflated false-positive rates. Proc. Natl Acad. Sci. USA 113, 7900-7905 (2016).

36. Xia, M., Wang, J. \& He, Y. BrainNet Viewer: a network visualization tool for human brain connectomics. PloS ONE 8, e68910 (2013).

37. Hosseini, S. M., Hoeft, F. \& Kesler, S. R. GAT: a graph-theoretical analysis toolbox for analyzing between-group differences in large-scale structural and functional brain networks. PloS ONE 7, e40709 (2012).

38. Tzourio-Mazoyer, N. et al. Automated anatomical labeling of activations in SPM using a macroscopic anatomical parcellation of the MNI MRI singlesubject brain. Neurolmage 15, 273-289 (2002).

39. Rubinov, M. \& Sporns, O. Complex network measures of brain connectivity: uses and interpretations. Neurolmage 52, 1059-1069 (2010).

40. Zhao, Y. J. et al. Brain grey matter abnormalities in medication-free patients with major depressive disorder: a meta-analysis. Psychol. Med. 44, 2927-2937 (2014).

41. Norton, D. J., McBain, R. K., Pizzagalli, D. A., Cronin-Golomb, A. \& Chen, Y. Dysregulation of visual motion inhibition in major depression. Psychiatry Res. 240, 214-221 (2016).

42. Furey, M. L. et al. Potential of pretreatment neural activity in the visual cortex during emotional processing to predict treatment response to scopolamine in major depressive disorder. JAMA Psychiatry 70, 280-290 (2013).

43. Bora, E., Fornito, A., Pantelis, C. \& Yucel, M. Gray matter abnormalities in Major Depressive Disorder: a meta-analysis of voxel based morphometry studies. J. Affect. Disord. 138, 9-18 (2012).

44. Kempton, M. J. et al. Structural neuroimaging studies in major depressive disorder. Meta-analysis and comparison with bipolar disorder. Arch. General. Psychiatry 68, 675-690 (2011).

45. Sacher, J. et al. Mapping the depressed brain: a meta-analysis of structural and functional alterations in major depressive disorder. J. Affect. Disord. 140, 142-148 (2012).

46. Eyre, H. A. et al. Altered resting-state functional connectivity in late-life depression: a cross-sectional study. J. Affect. Disord. 189, 126-133 (2016).

47. Guo, W. et al. Decreased insular connectivity in drug-naive major depressive disorder at rest. J. Affect. Disord. 179, 31-37 (2015).

48. Zou, K. et al. Abnormal functional connectivity density in first-episode, drugnaive adult patients with major depressive disorder. J. Affect. Disord. 194, 153-158 (2016).

49. Zhong, X., Pu, W. \& Yao, S. Functional alterations of fronto-limbic circuit and default mode network systems in first-episode, drug-naive patients with major 
depressive disorder: a meta-analysis of resting-state fMRI data. J. Affect. Disord. 206, 280-286 (2016).

50. Miller, C. H., Hamilton, J. P., Sacchet, M. D. \& Gotlib, I. H. Meta-analysis of functional neuroimaging of major depressive disorder in youth. JAMA Psychiatry 72, 1045-1053 (2015).

51. Zhang, X., Tang, Y., Zhu, Y., Li, Y. \& Tong, S. Study of functional brain homogeneity in female patients with major depressive disorder. Conf. Proc. IEEE Eng. Med. Biol. Soc. 2016, 2562-2565 (2016).

52. Liao, Y. et al. Is depression a disconnection syndrome? Meta-analysis of diffusion tensor imaging studies in patients with MDD. J. Psychiatry Neurosci. 38, 49-56 (2013)

53. Bonte, F. J. et al. Occipital brain perfusion deficits in children with major depressive disorder. J. Nucl. Med. 42, 1059-1061 (2001).

54. Jung, J. et al. Impact of lingual gyrus volume on antidepressant response and neurocognitive functions in major depressive disorder: a voxel-based morphometry study. J. Affect. Disord. 169, 179-187 (2014).

55. Truong, W. et al. Changes in cortical thickness across the lifespan in major depressive disorder. Psychiatry Res. 214, 204-211 (2013).
56. Maller, J. J. et al. Occipital bending in depression. Brain. 137(Pt 6), 1830-1837 (2014).

57. Liu, Y., Ho, R. C. \& Mak, A. Interleukin (IL)-6, tumour necrosis factor alpha (TNFalpha) and soluble interleukin-2 receptors (sIL-2R) are elevated in patients with major depressive disorder: a meta-analysis and meta-regression. J. Affect. Disord. 139, 230-239 (2012).

58. Pollak, Y. \& Yirmiya, R. Cytokine-induced changes in mood and behaviour: implications for "depression due to a general medical condition", immunotherapy and antidepressive treatment. Int. J. Neuropsychopharmacol. $\mathbf{5}$, 389-399 (2002).

59. Sugimura, T., Yoshimura, Y. \& Komatsu, Y. TNFalpha is required for the production of T-type $\mathrm{Ca}(2+)$ channel-dependent long-term potentiation in visual cortex. Neurosci. Res. 96, 37-44 (2015).

60. Hight, $\mathrm{K}$. et al. Time of day differences in the number of cytokine, neurotrophin, and NeuN-immunoreactive cells in the rat somatosensory or visual cortex. Brain Res. 1337, 32-40 (2010).

61. Mechelli, A., Friston, K. J., Frackowiak, R. S. \& Price, C. J. Structural covariance in the human cortex. J. Neurosci. 25, 8303-8310 (2005). 